\title{
Multiplication végétative in vitro de quelques espèces d'ormes
}

\author{
Noëlle DORION, P. DANTHU et C. BIGOT \\ avec la collaboration technique de B. GODIN, J. LEBCEUF, et M. CASENAVE \\ Ecole Nationale Supérieure d'Horticulture, \\ Chaire de Physiologie Végétale Appliquée, \\ 4, rue Hardy, F 78009 Versailles Cedex
}

\begin{abstract}
Résumé
La multiplication végétative in vitro a été mise au point sur plusieurs espèces d'ormes ( $U$. effusa Willd., $U$. campestris Mill., $U$. pumila L., $U$. americana L.) et clones, dits hybrides hollandais, dans le but de propager rapidement soit des sujets naturellement tolérants à la graphiose soit des sujets sélectionnés par hybridation ou par la voie des cultures cellulaires.

Les explants primaires (tigelles ou boutures de nœuds) sont, après enracinement avec ou sans AIB $0,5 \mathrm{mg} / 1$, multipliés par micropropagation. Deux méthodes ont été établies : l'une par microboutures de noeuds et d'apex $(7200$ plants/an), l'autre par décapitation et ramification axillaire ( 80 plants/an) quand la première est inefficace ( $U$. americana).

Les possibilités de multiplication dépendent des capacités d'enracinement ; on a observé : un effet clonal (nécessité ou non d'AIB $0,5 \mathrm{mg} / 1$ ), une influence de l'état de maturité du pied mère, une moindre aptitude des nœuds par rapport aux tigelles, et une augmentation du potentiel rhizogène au fil des repiquages in vitro.

La conservation in vitro, en chambre froide $\left(7^{\circ} \mathrm{C}, 7 \mathrm{~W} / \mathrm{m}^{2}\right.$ pendant $\left.8 \mathrm{~h}\right)$, a été possible pour des plantes enracinées (maximum 21 mois).

L'acclimatation en serre s'est effectuée sans difficulté $\left(18 / 20^{\circ} \mathrm{C}\right)$. Après 3 à 5 mois en serre, les jeunes plantes $(50 \mathrm{~cm})$ ont été installées en pépinière et se développent conformément à l'espèce.
\end{abstract}

\section{Introduction}

Toutes les espèces européennes et américaines appartenant au genre Ulmus sont sensibles à la graphiose (Dutch elm disease), maladie menaçant ce genre d'une disparition totale. A condition qu'elles soient sexuellement compatibles avec $U$. campestris, deux espèces auraient pu constituer une source de résistances transmissibles à l'orme champêtre, l'orme de Sibérie $(U$. pumila L.) et l'orme de Chine $(U$. parvifolia jacq.) Or, la première est attaquée, en France, par le champignon pathogène (Ceratocystis ulmi) ; quant à la seconde, sa floraison tardive (août-septembre) ne permet pas d'envisager un programme d'hybridation avec l'orme champêtre (sauf par un maintien temporaire en survie du pollen de l'une ou l'autre espèce). Cependant s'agissant de végétaux ligneux, l'existence d'une longue période de juvénilité avant la floraison est un handicap supplémentaire. 
On est donc conduit à exploiter plusieurs voies de recherche faisant intervenir les cultures in vitro pour:

- la multiplication en masse d'arbres naturellement tolérants ;

- la multiplication d'individus régénérés à partir de suspensions cellulaires soumises à l'action des toxines (Nordin et Strolele, 1981 ; Takai et Richards, 1978).

Dans les deux cas, la multiplication in vitro constitue la base d'une diffusion rapide pour les génotypes retenus. Toutefois le contrôle de la tolérance des plants provenant de culture in vitro est impératif avant toute diffusion. Les différents objectifs évoqués font l'objet d'études depuis trois ans au laboratcire.

La néoformation a déjà été obtenue dans le genre Ulmus. Ainsi, en 1975, DurzaN \& LopUShANSKI ont décrit les premiers l'obtention de jeunes plantes d'ormes américains en provenance de suspensions cellulaires établies à partir de cals d'hypocotyle. De même, Kannosky et al. (1982) ont aussi signalé la possibilité d'isoler de nombreux sujets à partir de cals hypocotylaires. Toutefois des méthodes mises au point à partir d'organes juvéniles ne s'appliquent pas obligatoirement à des tissus sélectionnés à l'état adulte. Notons enfin que l'établissement d'une callogénèse au cours de la multiplication (ce qui est signalé par Chalupa en 1979 pour $U$. campestris, U. effusa et $U$. scabra) peut avoir pour conséquence d'entraîner une hétérogénéité et l'apparition de déviants ayant perdu la caractéristique recherchée.

Cette note a pour objet de rapporter les conditions permettant de maîtriser sans callogénèse les étapes de la multiplication in vitro depuis le prélèvement de l'explant primaire (sur le terrain, en serre ou in vitro) jusqu'à l'élevage des plantes filles en serre et leur installation en pépinière. Les résultats présentés portent sur $U$. effusa Willd., $U$. americana $\mathrm{L}$., $U$. pumila $\mathrm{L}$. ainsi que $U$. campestris Mill. et ses hybrides naturels ou issus d'hybridations contrôlées tels que " Dodoens", "Commelin», "Plantijn » et «Groenveld » (clones dits hollandais).

\section{Matériel utilisé et techniques expérimentales}

Le matériel destiné à l'introduction in vitro est constitué :

- de graines provenant du Gurten kulm de: Berne, et récoltées soit sur un orme hybride (U.X. campestris Mill., noté OmBe). soit sur un orme diffus (U. effusa willd. noté OIBe) ; récoltées :

- de boutures de nœud lignifiées ou herbacées selon la période de prélèvement et

- en plein air à divers moments (hiver, été, automne), et occupant différentes positions sur l'arbre (cime, drageon, rejet de tronc ou de souche), pour $U$. campestris (OCBi, OCBa) et les clones hollandais (tabl. 2),

- en serre sur des rameaux en fin de croissance (U. americana OAmO et OAm xa4, campestris OCBa, et "Commelin») ;

- de pousses herbacées résultant de la croissance naturelle (printemps) ou d'un forçage en serre de boutures de nœud ( $U$. campestris OCBy et OCBi, pumila OPo', americana et hybrides hollandais. 
Les plantes mères, élevées en serre ( 2 à 3 ans), sont cultivées en conteneurs de 3 litres sur un substrat constitué de tourbe enrichie (TKS2 1/3), de terre franche $(1 / 3)$ et de sable (1/3), fertilisé par un engrais à libération lente (Type osmocote 15, 11, 15). Les plantes reçoivent un éclairage d'appoint de $14 \mathrm{~W} / \mathrm{m}^{2}$ (lampe Phytoclaude $400 \mathrm{~W}$ ) assurant une photopériode constante de $16 \mathrm{~h}$. La température maximale est de $28 \pm 4{ }^{\circ} \mathrm{C}$ en été, minimale de $18 \pm 2{ }^{\circ} \mathrm{C}$ en hiver. Les boutures de næuds, constituées d'un fragment de tige lignifiée et d'un bourgeon axillaire, sont placées pour le forçage en terrine contenant du sable désinfecté au cryptonol, et maintenues 15 à 30 jours en atmosphère confinée avant le prélèvement de la pousse herbacée provenant de chaque axillaire. Dans certains essais, les plantes mères et les pousses herbacées issues des boutures de nouds ont été traitées par une solution de Benlate $(600 \mathrm{mg} / \mathrm{l})$ en pulvérisation, $48 \mathrm{~h}$ avant le prélèvement.

\subsection{Introduction et culture in vitro}

Pour la désinfection, explants et samares fraîchement récoltés sont d'abord immergés $30 \mathrm{sec}$. dans l'alcool à $70^{\circ}$, puis 5 à $30 \mathrm{mn}$ selon la fragilité du matériel (20 $\mathrm{mn}$ pour les samares) dans une solution d'hypochlorite de calcium (50 à $100 \mathrm{~g} / \mathrm{l})$ additionnée de tween $80(100 \mathrm{ppm})$. Les explants sont ensuite rincés 3 fois dans l'eau distillée stérile. Après extraction, les graines sont introduites in vitro avec le seul tégument interne.

Les cultures ont été réalisées dans des boîtes de Pétri $(\varnothing 10 \mathrm{~cm})$, ou en tubes ( $\varnothing 22 \mathrm{~mm}$ ) obturés par des capuchons translucides de type Bellco.

Le milieu de base est constitué des macroéléments de Murashige et Skoog (1962) dilués au 1/2 ou au 1/4, des microéléments de HeLLER (1953) sans chlorure ferrique, de fer sous forme chélatée (Murashige et Skoog, 1962), des vitamines de Morel et WeTMOre (1951), et de saccharose $(10 \mathrm{~g} / \mathrm{l})$, auxquels ont été ajoutés $5,5 \mathrm{~g} / \mathrm{l}$ d'agar $\left(\right.$ OSI ${ }^{(1)}$. Sauf pour les graines, le milieu de base a été complété par une auxine (acide indolylacétique AIA, ou acide indolbutyrique AIB $0,5 \mathrm{ou} 1 \mathrm{mg} / \mathrm{l}$ ), ou une cytokinine (isopentényladénine 2 IP à $0,5 \mathrm{mg} / \mathrm{l}$ ou benzylaminopurine BAP à 0,3 et $0,5 \mathrm{mg} / \mathrm{l}$ ). Dans certains cas, du charbon actif ${ }^{(2)}$ a été apporté au milieu de culture $(2 \mathrm{~g} / 1)$ ainsi qu'un antibiotique (Rifampicine $50 \mathrm{ou} 100 \mathrm{mg} / \mathrm{l}$ ). Le $\mathrm{pH}$ a été ajusté à 5,5 avant autoclavage $\left(110^{\circ} \mathrm{C}, 20 \mathrm{~min}\right.$.). Les cultures ont été placées dans une chambre climatisée à la température de $25^{\circ} \mathrm{C}$ le jour et $22^{\circ} \mathrm{C}$ la nuit, en jours de $16 \mathrm{~h}$, sous une intensité lumineuse moyenne de $21 \mathrm{~W} / \mathrm{m}^{2(3)}$.

\subsection{Multiplication in vitro}

Deux méthodes de multiplication ont été utilisées : la première (fig. 1) consiste à bouturer les nœuds et les zones apicales; la seconde passe par l'entrée en croissance in situ des méristèmes axillaires préexistants sur les tigelles enracinées in vitro, par suppression de la dominance apicale (fig. 2) ; un gradient de vigueur décroissante apico-basal est noté dans ce dernier cas pour les ramifications. Ces dernières sont mises à leur tour en enracinement, puis traitées de la même manière.

(1) O.S.I. : Omnium Scientific International, Agar agar Ref. 1540.

(2) Charbon actif Merck. Ref. 2186.

(3) Eclairage fluorescent composé de tubes de $40 \mathrm{~W}$ en mélange : Lumière du jour (Philips). True Lite (Durotest), Grolux (Sylvania). 


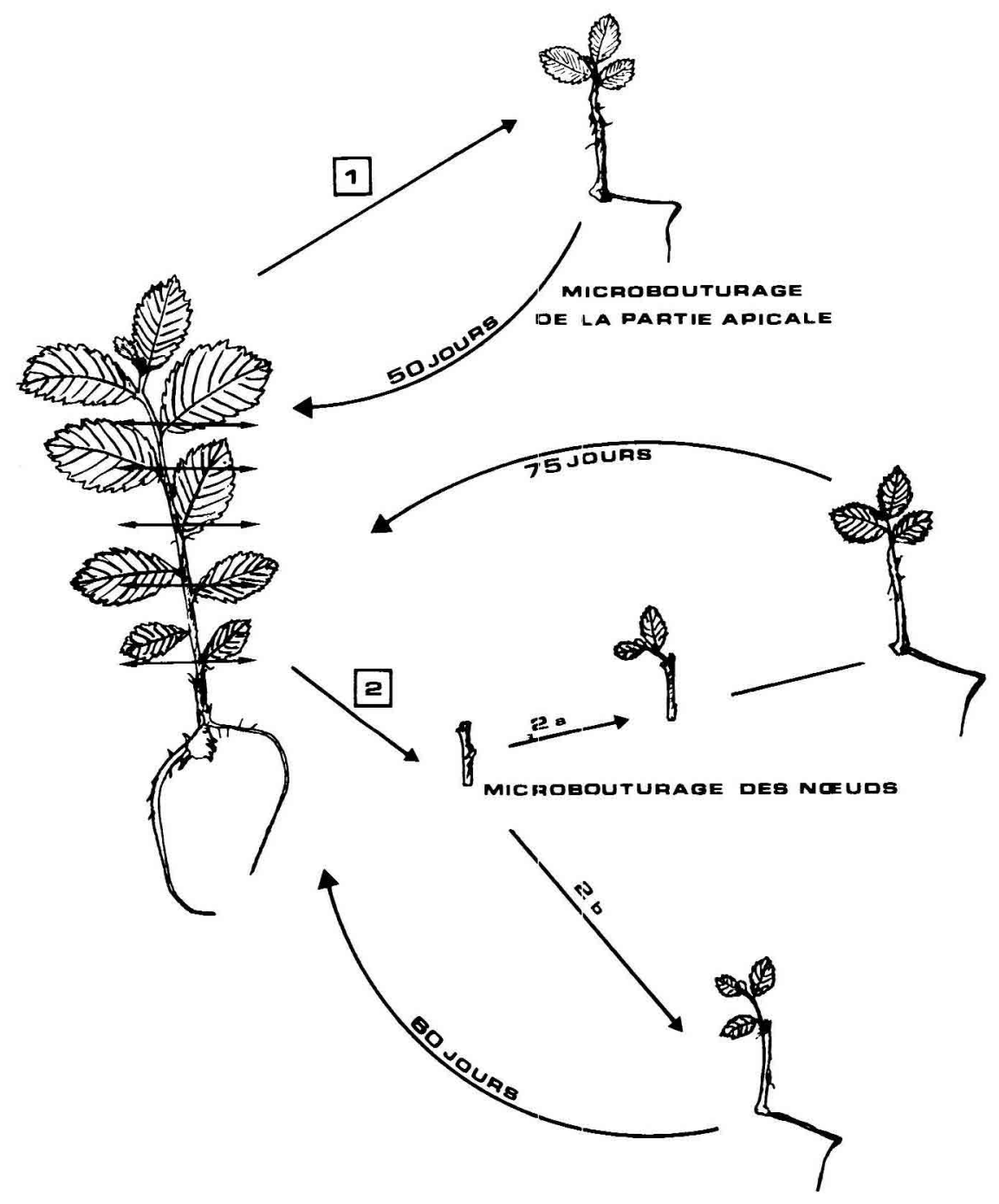

FIG. 1

Multiplication végétative in vitro par microbouture d'apex et de næuds. In vitro vegetative reproduction by micropropagation of the apex and nodes. 


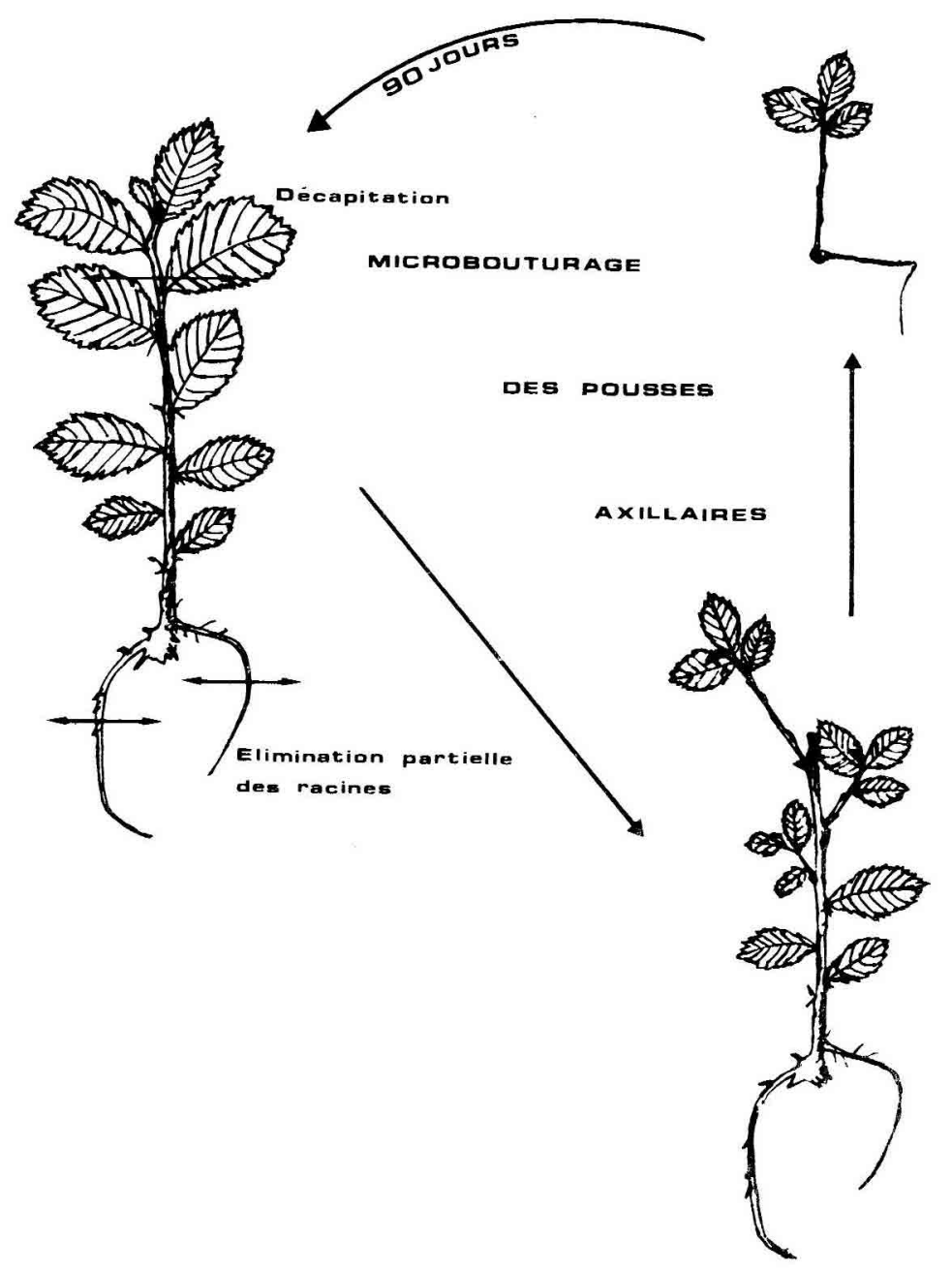

FIG. 2

Multiplication végétative in vitro par ramification des souches enracinées. In vitro vegetative reproduction by ramification of rooted shoots. 


\subsection{Conservation et levée de dormance}

Pour les essais de conservation, ou de levée de dormance, les plantes, à différents stades de développement, ont été installées pendant des temps variables dans une chambre froide $\left(7^{\circ} \mathrm{C}, 8 \mathrm{~h}\right.$ d'éclairement sous $\left.7 \mathrm{~W} / \mathrm{m}^{2}{ }^{(3)}\right)$, puis replacées pour les observations, en serre pour les plantes en pots, en chambre de culture pour les plantes in vitro.

\subsection{Acclimatation}

Les plantes enracinées in vitro, ont été extraites et placées dans des godets contenant de la tourbe enrichie $(1 / 2)$ et du sable (1/2). Elles ont d'abord été placées à $18 / 20^{\circ} \mathrm{C}$ en mini serre pendant 10 à 15 jours avec un éclairage d'appoint de $14 \mathrm{~W} / \mathrm{m}^{2}$ puis transférées à l'air libre.

\section{Résultats}

\subsection{Mise en culture}

\subsection{Désinfection}

Les contaminations exogènes et endogènes ont toujours considérablement limité l'introduction in vitro des explants; les pertes cobservées sont variables avec le type d'explant mis en culture, l'époque et le lieu de prélèvement (tabl. 1). Ainsi en plein air, la prise d'échantillons doit s"effectuer sur des pousses en croissance. Dans le cas de rameaux en fin de croissance, les contaminations sont toujours très importantes mais variables selon les capacités de croissance des axillaires. Ceux qui se développent activement peuvent être soustraits aux infections après élimination de l'explant primaire. Les prélèvements pratiqués sur du matériel préparé en serre (plante mère ou bouture de noud) donnent toujours de meilleurs résultats. Cependant, l'efficacité des traitements est fonction de la durée de désinfection. Pour «Commelin » par exemple, un traitement de $20 \mathrm{mn}$ dans l'hypochlorite de calcium à 7 p. 100 permet d'obtenir un taux d'infection inférieur à 5 p. 100 , alors qu'il atteint 33 p. 100 après un traitement de $10 \mathrm{mn}$. Toutefois, dans le cas des parties apicales de rameaux, la désinfection doit être limitée à $10 \mathrm{mn}$ pour éviter la nécrose des explants. Le pourcentage moyen d'infection est alors de 11 p. $100(7 / 66)$. Bien que l'addition de $100 \mathrm{mg} / \mathrm{l}$ de Rifampicine au milieu de culture limite les contaminations bactériennes (de 29 à 7 p. 100) quand le traitement par l'hypochlorite est court $(10 \mathrm{mn})$, le pourcentage d'infection varie peu puisqu'il diminue seulement de 42 à 24 p. 100.

Il apparait donc que le prélèvement des bourgeons axillaires sur des pieds mères cultivés en serre est la méthode qui présente le moindre risque de contamination.

\subsection{Croissance des explants primaires}

\subsection{Explants nodaux}

L'addition d'une auxine (AIA $0,5 \mathrm{mg} / 1$ ou AIB $0,5 \mathrm{mg} / \mathrm{l}$ ) et/ou d'une cytokinine (2 IP $0,5 \mathrm{mg} / \mathrm{l}$; BAP 0,3 ou $0,5 \mathrm{mg} / 1$ ) ne stimule pas la croissance des pousses 
TABLEAU 1

Perte par contamination (p. 100) observée selon le type d'explant, l'époque et le lieu de prélèvement.

Loss by contamination (p. 100) observed depending on the type of explant, season and sample location.

\begin{tabular}{|c|c|c|c|c|}
\hline \multirow{2}{*}{ Type d'explant } & \multirow{2}{*}{ Stade } & \multicolumn{2}{|c|}{ Prélèvement } & \multirow{2}{*}{$\%$ contaminations } \\
\hline & & Epoque & Lieu & \\
\hline \multirow{3}{*}{$\begin{array}{l}\text { Bourgeons } \\
\text { axillaires }\end{array}$} & Rameau ligneux & Automne & Plein air & 100 p. 100 \\
\hline & $\begin{array}{l}\text { Rameau en fin } \\
\text { de croissance }\end{array}$ & Eté & Plein air & 80 à 100 p. 100 \\
\hline & $\begin{array}{l}\text { Rameau en fin } \\
\text { de croissance }\end{array}$ & & Serre & 0 à 40 p. 100 \\
\hline \multirow[t]{2}{*}{$\begin{array}{l}\text { Pousses } \\
\text { feuillées }\end{array}$} & $\begin{array}{l}\text { Croissance } \\
\text { naturelle }\end{array}$ & Printemps & Plein air & 25 p. 100 \\
\hline & Croissance forcée & Hiver & Serre & 16 à 70 p. 100 \\
\hline
\end{tabular}

préformées dans les bourgeons. De plus, les combinaisons auxine-cytokinine, de même que les cytokinines utilisées seules, entraînent une callogénèse active au niveau des sections ; l'axillaire est alors rapidement recouvert par les nouveaux tissus et inhibé. En présence d'auxine aucun cal n'est observé, et on note une légère inhibition puisque 16 p. 100 des bourgeons s'allongent en présence d'AIB $(0,5 \mathrm{mg} / \mathrm{l})$ et $22 \mathrm{p} .100$ en présence d'AIA $(0,5 \mathrm{mg} / \mathrm{l})$ contre 34 p. 100 sans régulateur. Lorsque l'axillaire reste inhibé ou présente un débourrement tardif, (76 p. 100 des bourgeons dans le cas de «Commelin »), on observe à la partie supérieure de la tige la néoformation de pousses au niveau du cambium (fig. 3). Ces pousses (1,4 en moyenne par explant) pourraient être utilisées pour augmenter la quantité de tigelles introduites en multiplication dans la mesure où, issues de néoformations, leurs caractéristiques physiologiques (tolérance à la graphiose, en particulier dans le cas de "Commelin ") ou morphodynamiques ne seront pas modifiées.

\subsection{Pousses herbacées}

A partir d'extrémités apicales feuillées (apex +1 à 2 feuilles développées), la croissance est dépendante de l'aptitude de chaque clone à l'enracinement (capacité génétique + capacité ontogénique déterminée par l'âge du pied mère). Ainsi, les 2 clones d' $U$. americana introduits in vitro s'enracinent sans apport d'auxine, de même que quelques apex du clone OCBi. Toutefois, les explants de ces deux espèces provenaient soit d'arbres jeunes (2 à 3 ans) soit de rejets de souches qui pourraient présenter des potentialités juvéniles (Franclet, 1981). Les clones hollandais «Vegeta » et «Plantijn " (âge > 5 ans) s'enracinent aussi sans régulateur de croissance, en 10 jours. Par contre, la rhizogénèse est plus difficile à obtenir dans le cas de "Commelin "; après un mois, le taux de réussite est seulement de 43 p. 100 (17/40) en présence d'AIB $0,5 \mathrm{mg} / \mathrm{l}$. 


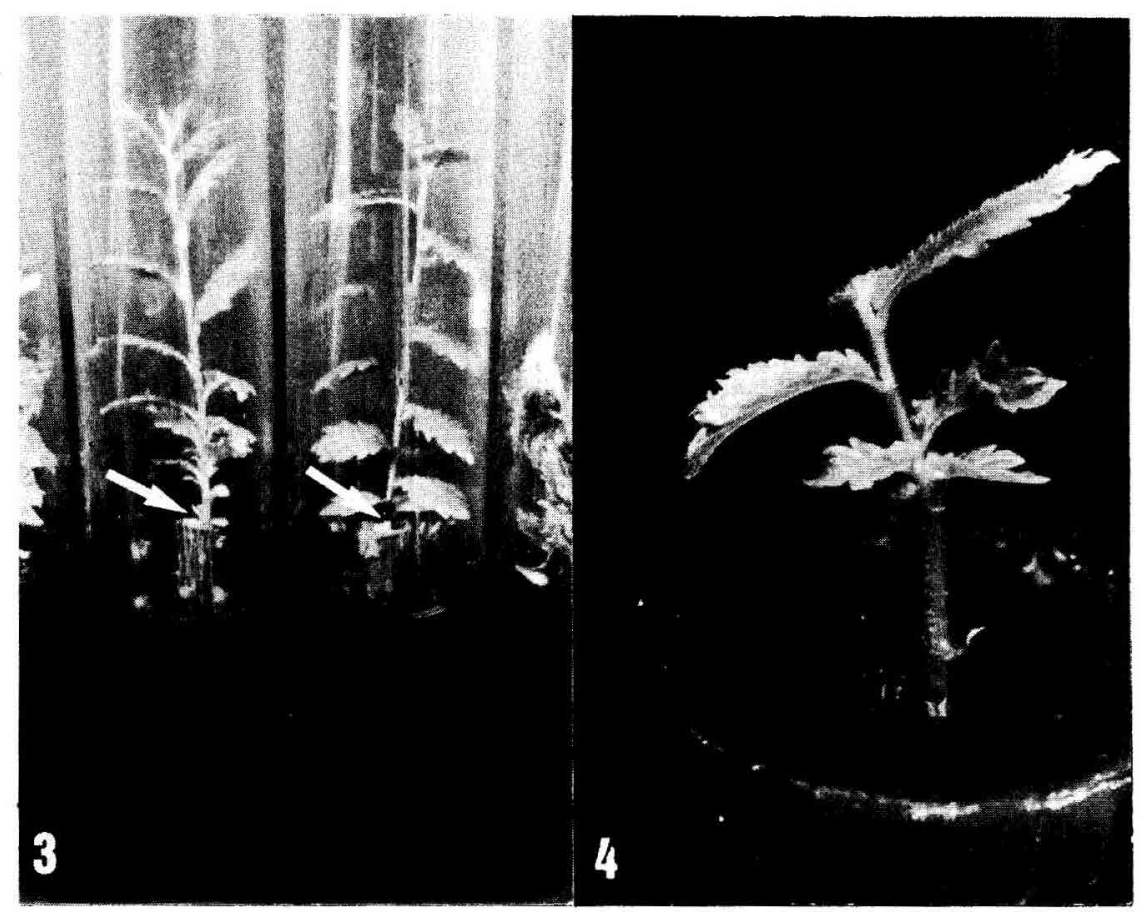

Fig. 3

Aspect des pousses néoformées au niveau du cambium $(\rightarrow)$ : clone OCBa.

Characteristics of neoformed shoots at the cambium level : clone OCBa.

Fig. 4

Entrée en croissance des bourgeons axillaires sur boutures de næud (OCBa) après 28 jours.

Beginning of growth of the axillary buds on nodal cuttings (OCBa) after 28 days.

\subsection{Micropropagation in vitro}

Les méthodes de multiplication ont été mises en place à partir des sujets enracinés issus des manipulations précédentes ou de germinations in vitro.

Les tentatives pour obtenir une méthode de multiplication par développement d'axillaires ont échoué en raison d'une callogénèse intense à la base des explants due à la présence de la cytokinine (BAP $0,5 \mathrm{mg} / 1$ ). Seule une multiplication par fractionnement de plantules (fig. 1 et 2) a donc été établie.

\section{Parties apicales}

L'enracinement est favorisé par la dilution des macroéléments (MS/4) et dans certains cas par un apport de charbon actif $(\hat{z}, \mathbf{g} /:)$. On a pu noter en outre (tabl. 2$)$ :

- un effet spécifique : 90 p. 100 d'enracinement pour $U$. pumila contre 58 p. 100 pour un $U$. campestris de même âge ; 


\section{TABleau 2}

Influence de divers facteurs sur l'enracinement après 40 jours de quelques clones d'ormes cultivés in vitro depuis plus d'un an (essais réalisés sur des effectifs $>12$ ).

The influence of various factors after 40 days, on the rooting of some elm clones cultivated in vitro for more than a year (trial carried out on a population $>12$ ).

\begin{tabular}{|c|c|c|c|c|}
\hline Espèce et clone & Origine & AIB $\mathrm{mg} / \mathrm{l}$ & $\begin{array}{c}\text { Culture } \\
\text { in vitro }\end{array}$ & Enracinement \% \\
\hline \multicolumn{5}{|l|}{ U. campestris } \\
\hline OCBa . & $\begin{array}{l}\text { Rejets de tronc } \\
1 \text { an }\end{array}$ & 0 & 2 ans & 60 \\
\hline OCBi & $\begin{array}{l}\text { Rejets de souche } \\
2 \text { à } 3 \text { ans }\end{array}$ & AIB 0,5 & 1 an & 58 \\
\hline OCBy $\ldots$ & $\begin{array}{l}\text { Drageons } \\
3 \text { à } 5 \text { ans }\end{array}$ & AIB 0,5 & 2 ans & 13 \\
\hline \multicolumn{5}{|l|}{ U.X. campestris } \\
\hline OmBe $\mathbf{m} \ldots \ldots$ & Graine & 0 & 1 an & 100 \\
\hline OmBe $k \ldots \ldots$ & Graine & 0 & 1 an & 90 \\
\hline OmBe $1 \ldots \ldots$ & Graine & AIB 0,5 & 1 an & 71 \\
\hline \multicolumn{5}{|l|}{ U. effusa } \\
\hline OlBe $\mathrm{f}$ & Graine & 0 & 1 an & 88 \\
\hline OlBe g . . & Graine & AIB 0,5 & 1 an & 50 \\
\hline U. pumila Opo' & Arbuste $4 / 5$ ans & 0 & 1 an & 90 \\
\hline \multicolumn{5}{|l|}{$\begin{array}{l}\text { Hybrides } \\
\text { hollandais }\end{array}$} \\
\hline «Dodoens».. & Arbuste $>3$ ans & AIB 0,5 & 1 an & 73 \\
\hline «Dodoens » & Arbuste $>3$ ans & 0 & 1 an & 9 \\
\hline «Vegeta " . & Arbuste $>3$ ans & 0 & 1.5 an & 94 \\
\hline «Commelin » & Arbuste $>3$ ans & 0 & 1,5 an & 87 \\
\hline
\end{tabular}

$\left(^{*}\right)$ Culture in vitro : temps passé en multiplication (6 à 7 subcultures/an).

- un effet clonal pour $U$. campestris l'enracinement est de $13,58,60$ p. 100 , selon le clone considéré ;

- un effet de l'état de maturité des pieds mères, puisque pour $U$. campestris et hybrides ( $U . X$. campestris), les clones issus de germination in vitro se sont mieux enracinés (71 à 100 p. 100) que ceux issus des drageons et rejets (13 à 60 p. 100);

- un effet des régulateurs de croissance. Ainsi 7 clones se sont enracinés spontanément, pour les 5 autres l'AIB a facilité la rhizogénèse. Ce résultat est particulièrement net pour « Dodoens ».

Enfin on a noté une augmentation importante de laptitude au bouturage au fur et à mesure des cycles de multiplication in vitro. Ainsi pour OCBa, sans régulateur de croissance, le p. 100 d'enracinement à 50 jours passe de 50 à 100 entre 1 et 3 ans de culture in vitro (6 à 7 subcultures par an). De même pour "Commelin ", ce pourcentage s'accroît de 42 à 87 p. 100 entre 2 et 18 mois et l'exigence en AIB disparait. 
Les plantes enracinées, issues du bouturage des parties apicales, ont été réintroduites dans le cycle de multiplication ou passées en pépinière après une phase d'acclimatation en serre ( $>5$ mois).

\subsection{Microbouturage des nouds isolés}

Deux phénomènes ont été pris en compte : l'allongement du méristème axillaire, et l'enracinement du nœud porteur.

L'entrée en croissance des axillaires se manifeste pour 50 à 90 p. 100 des nouds, 20 à 30 jours après l'isolement (fig. 4), l'élongation se poursuit activement après l'enracinement du nœud porteur. L'allongement moyen après 46 jours pour OCBa est de $31,8 \pm 11,8 \mathrm{~mm}$ pour les nœuds enracines, contre $11,7 \pm 5,1 \mathrm{~mm}$ pour les non enracinés. Les pousses allongées sont réinsérées directement dans un cycle de multiplication après 60 jours environ. Pour les axillaires des nœuds non enracinés, la croissance est obtenue après séparation de l'axe porteur et microbouturage. On a en effet observé que la rhizogénèse est plus facile à obtenir sur les parties apicales que sur les nœuds pour lesquels l'addition d'AIB $0,5 \mathrm{mg} / 1$ est le plus souvent nécessaire (tabl. 3).

Tableav 3

Comparaison de l'aptitude à l'enracinement des apex et des nouds isolés après 40 jours de microbouturage at un an de culture in vitro.

A comparison of rooting aptitude for the apex and isolated nodes after 40 days of micropropagation and one year of in vitro culture.

\begin{tabular}{|c|c|c|c|}
\hline \multirow{2}{*}{ Clone } & \multirow{2}{*}{$\begin{array}{c}\begin{array}{c}\% \text { d'enracinement } \\
\text { des parties apicales }\end{array} \\
\text { AIB } 0 \mathrm{mg} / \mathrm{l}\end{array}$} & \multicolumn{2}{|c|}{$\%$ d'enracinement des nœuds } \\
\hline & & $\mathrm{AIB} \quad 0 \mathrm{mg} / \mathrm{I}$ & AIB $0,5 \mathrm{mg} / \mathrm{l}$ \\
\hline OCBa $\ldots \ldots \ldots$ & 60 & 9 & 33 \\
\hline $\mathrm{OmBe} \mathrm{m}$ & 100 & 17 & 71 \\
\hline OmBe k & 90 & 22 & 31 \\
\hline OlBe $f$. & 88 & 17 & 75 \\
\hline Opo' & 90 & 71 & 42 \\
\hline
\end{tabular}

TABLEAL 4

Evolution de l'aptitude à l'enracinement in vitro, au cours du temps, chez deux clones OCBa et OmBe m.

Changes in the rooting aptitude in vitro, with time, for two clones, OCBa and OmBe m.

\begin{tabular}{|c|c|c|c|c|}
\hline \multirow{2}{*}{ Clone } & \multirow{2}{*}{$\begin{array}{l}\text { Temps passé } \\
\text { en multiplication }\end{array}$} & \multirow{2}{*}{$\begin{array}{l}\text { Temps depuis } \\
\text { le microbouturage }\end{array}$} & \multicolumn{2}{|c|}{$\%$ d'enracinement } \\
\hline & & & AIB $0,5 \mathrm{mg} / 1$ & AIB $0 \mathrm{mg} / \mathrm{l}$ \\
\hline \multirow[t]{3}{*}{$\mathrm{OmBe} \mathrm{m} \ldots$} & 12 mois & 40 jours & 71 & 17 \\
\hline & 36 mois & 30 jours & 55 & 83 \\
\hline & 36 mois & 40 jours & 91 & $10)$ \\
\hline \multirow[t]{2}{*}{$\mathrm{OCBa}$} & 10 mois & 50 jours & 54 & 70 \\
\hline & 36 mois & 50 jours & 100 & 83 \\
\hline
\end{tabular}


Dans le cas de «Dodoens ", l'enracinement des nouds reste faible: 14 p. 100 même en présence d'AIB ; toutefois, on peut aussi constater que plus le nombre de cycles de multiplication in vitro augmente, plus les nœuds s'enracinent facilement (tabl. 4).

\section{Efficacité de la multiplication}

En associant le microbouturage des parties apicales et des nœuds, on peut, en un an environ, espérer obtenir à partir d'une tigelle, $7200\left(4,4^{6}\right)$ plantules enracinées si on retient les conditions suivantes :

- Enracinement des parties apicales : 100 p. 100 ;

- Débourrement des nœuds : 90 p. 100 ;

- Enracinement des nouds : 80 p. 100.

Toutefois ces chiffres ne peuvent être obtenus pour certains clones, en particulier $U$. americana (OAmO et OAm xa4) ainsi que "Plantijn" et "Groenveld " pour lesquels le développement des axillaires est faible ou nul sur nœud isolé dans nos conditions d'expériences. Dans ce cas les plantes in vitro peuvent être décapitées pour lever la dominance apicale, puis repiquées avec leur système racinaire (fig. 2). Il est alors possible d'obtenir en 40 jours, en moyenne, environ 2,5 ramifications par plante de 7 à 8 nœuds ; l'addition d'une cytokinine (2 IP $0,5 \mathrm{mg} / \mathrm{l})$ n'améliore pas l'intensité de la ramification. Selon le schéma proposé (fig. 2), le rendement théorique de la multiplication n'est dans ce cas que de $81\left(3^{4}\right)$ plantes enracinées par an, ce mode de propagation est donc beaucoup moins efficace que le précédent. Toutefois, on peut envisager de passer progressivement au procédé de microbouturage de nœuds si comme on l'a vu par ailleurs la réactivité des explants augmente au cours de la culture in vitro.

\subsection{Conservation des plantes in vitro}

La constitution d'un conservatoire de génotypes implique de limiter la fréquence des repiquages, manipulations difficiles à assurer pour un grand nombre de clones, et pouvant conduire à des variations non contrôlées. Quelques résultats préliminaires sur la conservation en chambre froide ont été obtenus, bien que les effectifs mis en expériences soient faibles. On a pu noter que pour des plants mis au froid après enracinement la survie est de 21 mois environ. 7 à 10 jours après le retour dans la chambre de culture, un repiquage avec une multiplication par bouture de nouds peut être pratiqué. Pour les clones les plus résistants (OCBa et OCBi 100 p. 100 de survie) le coefficient de multiplication a été de 4 à 5 après 18 mois. Si les plantes ne sont pas enracinées au moment de la mise au froid, létat dormant paraît souhaitable ; cependant la conservation ( "Dodoens ») n’a pas été prolongée au-delà de 9 mois : en effet les bourgeons commencent leur croissance en chambre froide; il est alors nécessaire de les repiquer pour éliminer la partie basale lignifiée, toutefois dans ce cas les tigelles herbacées sont trop courtes pour être multipliées.

\subsection{Acclimatation et croissance}

L'acclimatation des plants issus de culture in vitro a été effectuée sans difficulté dans les conditions décrites plus haut. Deux facteurs importants interviennent dans la reprise de croissance : le stade de développement au passage en serre et l'effet clonal.

Lorsque les plantes sont acclimatées pendant une phase d'arrêt de croissance (formation d'initium foliaire sans allongement), l'acclimatation est possible, mais les 


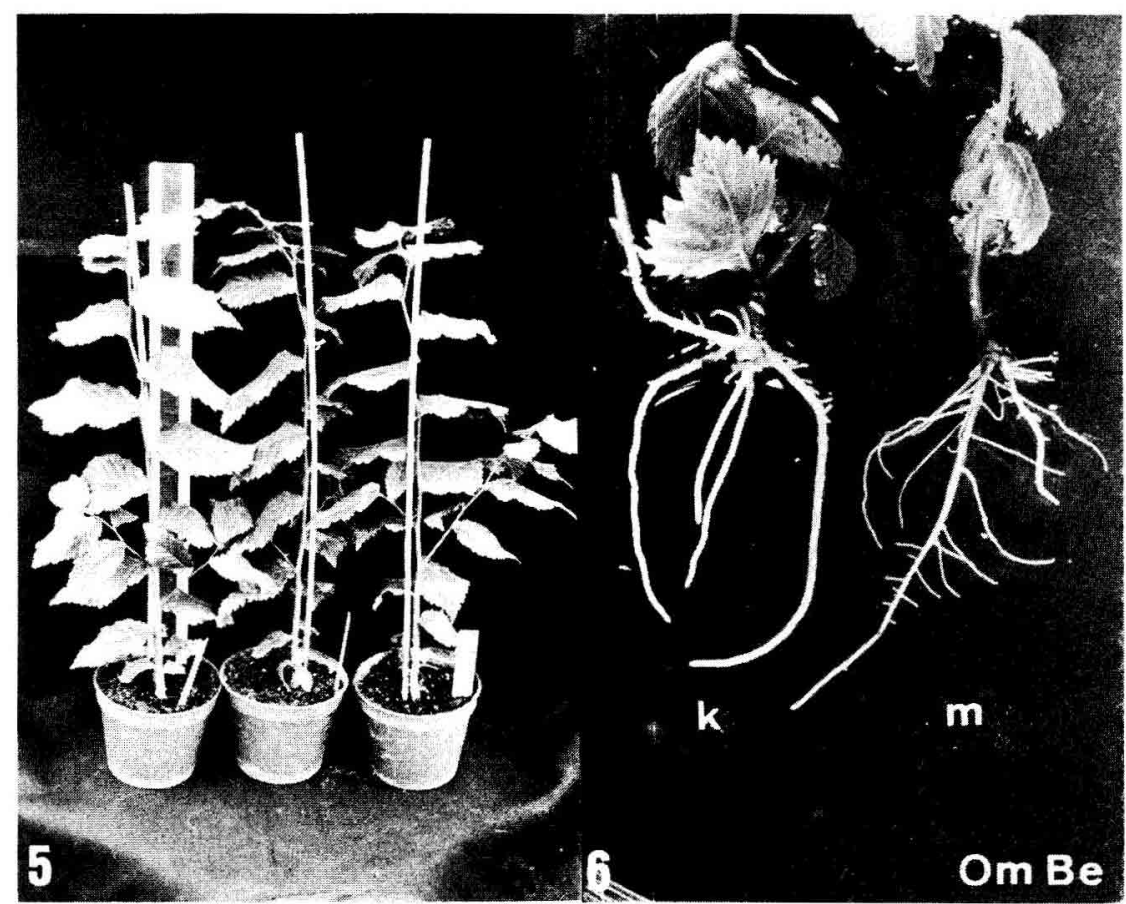

FIG. ;

Plantes $d u$ clone OmBe $k$ après acclimatation en serre $(5$ mois). Clone OmBe $k$ plants after acclimatization under glass (5 month).

FIG. 6

Aspect des systèmes racinaires de deux clones d'U. X campcstris issus de 2 graines prélevées sur le même arbre et multipliés in vitro $(\mathrm{OmBe} m$ et k).

Character of the rooting systems of two clones of $\mathrm{U}$. X campestris coming from 2 seeds gathered from the same tree and reproduced in vitro $(\mathrm{OmBe} m$ and $k)$.

plantes entrent rapiỏement en repos (f́rmation d'un bourgeon terminal avec écailles) même en jours longs. Cet état s'est maintenu pendant 6 mois. Un passage de 2 mois en chambre froide permet la reprise d'élongation dans la semaine qui suit le retour à $20^{\circ} \mathrm{C}$.

Lorsque les plantes sont acclimatées en phase: de croissance active, l'allongement se manifeste rapidement ( 15 jours). Après 3 à 5 mois les plantes atteignent une hauteur moyenne de $50 \mathrm{~cm}$ (fig. 5). Toutefois, on peut observer quelques variations suivant les clones. Ainsi, le clone $\mathrm{OmBe} \mathrm{m}$ présente, après 1 mois $1 / 2$, une croissance moyenne de $30,33 \pm 3,50 \mathrm{~cm}$, deux fois plus importante que e clone OmBe $\mathrm{k}(17,0+2,4 \mathrm{~cm})$. Ce résultat pourrait être en corrélation avec le type d'enracinement de chacun des clones ; en effet OmBe $m$ présente un système racinaire fasciculé, très ramifié, alors que celui de $O \mathrm{mBe} k$ est formé de quelques racines peu ramifiées à tendance callogène (fig. 6). Quelques sujets issus de culture in vitro (OCBi) ont été installés en pépinière et poursuivent depuis 3 ans un développement apparemment normal. 


\section{Discussion}

Deux méthodes de multiplication in vitro ont été établies; la première utilisant le fractionnement de sujets enracinés est envisageable pour les clones présentant une forte réactivité dès l'introduction in vitro ; la seconde utilisant l'aptitude à la ramification des plantules décapitées, beaucoup moins efficace, est applicable aux clones à réactivité faible, pour lesquels les nœuds isolés ne présentent, dans les conditions de culture utilisées, ni croissance des méristèmes axillaires, ni enracinement. Ces méthodes de multiplication provenant exclusivement du développement de méristèmes préformés, ne devraient pas conduire à un pourcentage de variation supérieur à celui obtenu par les méthodes de multiplication végétative traditionnelles.

Il faut noter que ces deux méthodes excluent l'utilisation de cytokinines pour stimuler le développement des axillaires, alors que ce procédé est employé efficacement pour d'autres espèces ligneuses comme par exemple le merisier (RIFFAud et CoRnu, 1981). Dans le cas des ormes une prolifération intense de cals est induite par les cytokinines, tant au niveau de l'introduction des explants primaires in vitro que dans la phase de multiplication. Chalupa (1979) rapporte des résultats analogues sur plusieurs espèces d'ormes avec des doses de BAP de 0,1 à $2 \mathrm{mg} / \mathrm{l}$.

En ce qui concerne l'utilisation d'auxines en vue de stimuler la rhizogénèse (en particulier AIB $0,5 \mathrm{mg} / \mathrm{l}$ ), la réaction des explants dépend à la fois :

- du génome puisque les clones étudiés présentent des exigences vis-à-vis de l'AIB très nettement différentes dans le cas de matériel juvénile (issu de germination), comme cela a été observé par exemple avec les Vaccinium (FISCHER-DazY et al., 1984);

- de l'état physiologique, puisqu'on a pu mettre en évidence :

1) une exigence différente vis-à-vis de l'auxine pour des boutures de nœud utilisées soit en culture primaire, soit en repiquage in vitro. Dans le premier cas, les axillaires peuvent se développer sans rhizogénèse à partir de l'explant et l'auxine n'est pas nécessaire, contrairement aux observations faites sur Gardenia par exemple (Dumanols et al., 1984). Dans le second cas, l'allongement de l'axillaire est consécutif à l'enracinement qui nécessite le plus souvent la présence d'AIB. Il est probable que le niveau de réserves des explants (éléments nutritifs et régulateurs) est la cause principale de ces différences ;

2) une modification des capacités initiales de rhizogénèse au cours de la multiplication, l'auxine devenant inutile après quelques repiquages. Ce phénomène peut s'expliquer par un effet rajeunissant des cultures in vitro (Boxus, 1978 ; ZIMMERMANN, 1980) qui se traduit par une augmentation du potentiel d'enracinement (SRISKANDARAJAH et al., 1982 ; Dumanois et al., 1984).

Actuellement, les méthodes de multiplication végétative rapportées dans cet article ont déjà permis :

- une mise en conservatoire de 19 clones dont certains (5 clones hollandais) présumés plus tolérants à la graphiose ;

- une multiplication intensive de certains clones (établissement de pied-mères in vitro).

Toutefois, ces méthodes pourraient devenir plus performantes dès la phase d'introduction in vitro des explants primaires, soit par une meilleure maîtrise des conditions de prélèvement, soit par un accroissement du nombre des tigelles à l'origine des cycles de 
multiplication. L'utilisation des pousses néoformées à partir du cambium des tiges sectionnées, pourrait être aussi un moyen efficace d'augmenter la quantité initiale de plantules. Il faut noter que cette potentialité des explants primaires a été rapportée depuis longtemps par GaUtheret (1940a et b) sur des fragments de cambium isolé, et par JaCQUiot $(1949,1977)$ au niveau de la portion cambiale d'explants plus complexes (tige ou cambium + bois et liber) soustraits à la dominance de la cime. Toutefois, cette possibilité n'est réellement intéressante que dans la mesure où les tigelles néoformées présentent un développement conforme à la plante mère.

Reçu le 3 juillet 1986.

Accepté le 17 mars 1986.

\section{Remerciernents}

Les auteurs remercient vivement M. Pinon (C.R.F. Nancy) pour leur avoir fourni le matériel nécessaire à l'introduction in vitro des variétés hollandaises sélectionnées par le $\mathrm{P}^{\mathrm{r}}$ Heybroek, et M. Cornu (INRA, Orléans) pour les exemplaires d $\left.\right|^{\top} U$. pumila et $U$. americana.

Les auteurs remercient également E. Nawoj pour la mise en page de ce travail.

\section{Summary}

The in vitro reproduction of some elm species

Because of their vulnerability to Dutch elm disease. European and American elms are in danger of disappearing. Also, improvement of tolerance by hybridisation is delicate (because of the rarity of resistant genotypes, the necessity for inlerspecific crosses, and the length of the juvenile period).

The use of in vitro cultures allows:

- mass reproduction of naturally tolerant trees ;

- the reproduction of individuals regenerated in cellular suspensions resistant to the toxins of the pathogen (Ceratocystis ulmi).

In this case a method of in vitro vegetative reproduction was perfected on clones of various elms derived from many species (u. effusa, pumila. americana, campestris) and from Dutch hybrids (Dodoens Vegeta, Plantijn, and Groenveld).

\section{Growing conditions}

The cultural medium for reproduction, consisted of the diluted macroelements of Murashige and Skoog (MS/2 and MS/4), the microelements of Heller, chelated iron (Fe EDTA), the vitamins of Morel and Wetmore, saccharose $10 \mathrm{~g} / \mathrm{l}$. and also active charcoal $2 \mathrm{~g} / 1$.

Depending on the trial, the medium was completed by the addition of an auxin (IAA, IBA, 0.5 or $1 \mathrm{mg} / 1$ ) or a cytokinin (2 I P $0.5 \mathrm{mg} / \mathrm{l}$ or BAP 0.3 and $0.5 \mathrm{mg} / 1$ ). The cultures were placed in a controlled environment chamber $\left(25^{\circ} \mathrm{C}\right.$ Day $/ 22^{\circ} \mathrm{C}$ Night $)$ with 16 hour days $\left(21 \mathrm{~W} / \mathrm{m}^{2}\right)$.

\section{Cultures}

Contamination is a limiting factor in in vitro development. The best results were obtained either by sampling herbaceous shoots growing in the open air or after forcing under glass, or using the explants (apical parts or nodal cuttings) coming from the parent stool grown under glass. Sterilisation with $\mathrm{Ca}$ hypochlorite for 10 to 20 minutes, combined with a treatment of the parent stool with Benlate $(600 \mathrm{mg} / 1) 48$ hours before sampling, limited infection to 5 or $11 \mathrm{p}$. 100 . 
The axillary growth of the nodal cuttings develop without growth regulators, the cytokinins produce an intense callusing which cannot be used. When the development of the axillaries is slow (76 p. 100) the neoformed shoots (1.4/explant) begin to grow at the cambium level.

The herbaceous shoots are mainly rooted without growth regulators. For certain clones like "Commelin », rooting was moderate (43 p. 100) even using IBA $0.5 \mathrm{mg} / \mathrm{l}$.

In vitro micropropagation

As soon as the samples have rooted following the above manipulations, or in vitro germination, two reproductive systems were established: the first, by splitting the plantlets, and the second by a ramification of the decapitated plantlets for the clones which produced necrotic nodes after isolation.

Elongation of the shoots, indispensable for reproduction, depends on the rooting of the explant. With reference to this subject one can observe :

- a clonal effect, 7 clones rooted spontaneously, for the others an addition of IBA is beneficial ;

- an influence of the level of maturity of the parent stools (a juvenile effect) ;

- an aptitude towards root formation, less for the nodes than for the apical parts ;

- an amelioration of rooting capacity during in vitro reproduction.

\section{Maintenance of plants in vitro}

Results were obtained for the clones maintained at $7^{\circ} \mathrm{C}$ with 8 hours of light $\left(7 \mathrm{~W} / \mathrm{m}^{2}\right)$. After rooting, the plants were maintained for 21 months. The first reproduction was carried out 7 to 10 days after returning to $25^{\circ} \mathrm{C}$. Maintenance was also possible (9 months) for the non-rooted tigelles with a dormant terminal bud.

\section{Acclimatization and growth}

Acclimatization was carried out in mini-glasshouses at $18 / 20^{\circ} \mathrm{C}$ for 10 to 15 days. If growth stopped, treatment for 2 months in a cold chamber was necessary to break the inhibition. The mean elongation obtained was from $50 \mathrm{~cm}$ after 4 months, but varied according to the clones and the state of their rooting systems. After planting, the elms from in vitro culture seemed to develop normally.

The methods perfected allowed us to obtain about 7.200 (for the first) and 81 (for the second) rooted plantlets per year, without the risk of variation (absence of callus).

They have already been used for :

- the conservation of sensitive, or more or less tolerant clones (the Dutch clones) ;

- the stockage of parent stools for the preparation of cellular suspensions.

However, the phase of introduction in vitro still needs to be improved. The use of neoformed shoots on cambium could be envisaged to increase the number of the initial plantlets, if the system would not generate variations.

\section{Références bibliographiques}

Boxus P., 1978. In vitro multiplication of woody species. Publié par la station des cultures fruitières et maraîchères. Chaussée de Charleroi 234, 5800 Gembloux, Belgique, 300 p.

Chalupa Y., 1979. In vitro propagation of some broad-leaved forest trees. Comm. Inst. Forest. Cechosiov., 11, 159-170.

Dumanois C., Godin B., Bigot C., 1984. Multiplication végétative in vitro de Gardenia jasminoides Ellis. J. Plant Physiol., 116, 389-407.

Durzan D.J., Lopushanski S.M., 1975. Propagation of American elm via cell suspension cultures. Can. J. For. Res., 5, 273-277.

Fischer-Daty A.C., Godin B., Bigot C., 1984. Variabilité des capacités organogènes in vitro observées à partir de matériel juvénile dans le cas de quelques myrtilliers américains (Vaccinium sp.). 4" Colloque sur les recherches fruitières, Bordeaux, 63-75.

Franclet A., 1981. Rajeunissement et propagation végétative des ligneux. Ann. Rech. Sylv., 1140 . 
Gavtheret R., 1940a. Recherches sur le bourgeonnement du tissu cambial d'Ulmus campestris cultivé in vitro. C.R. Acad. Sci., Paris, 210, 632-634.

GAUTHERET R., 1940b. Nouvelles recherches sur le bourgeonnement du tissu cambial d'Ulmus campestris cultivé in vitro. C.R. Acad. Sci., Paris, 210, 744-746.

Hel.ter R., 1953. Recherches sur la nutrition minérale des tissus végétaux, cultivées in vitro. Ann. Sci. nat., Paris, 14, 1-223.

JaCQuiot C., 1949. Observations sur la néoformation de bourgeons chez le tissu cambial d'Ulmus campestris cultivé in vitro. C.R. Acad. Sci., Paris, 229, 529-530.

Jacquiot C., 1977. Néoformations de bourgeons à partir de cambium d'Ulmus campestris et d'autres espèces. Bull. Soc. Bot. France, 124, 141-143.

Karnosky D.F., Mickler R.A., Lange D.D., 1982. Hormonal control of shoot and root induction in hypocotyl callus cultures of american elm. In viro, 18, 275.

Morel G., Wetmore R.H., 1951. Fern callus tissue culture. Am. J. Bot., 38, 141-143.

Murashige T., Skoog F., 1962. A revised medium for rapid growth and bioassays with tobacco tissue culture. Physiol. Plant., 15, 473-497.

Nordin J.H., Strobel G.A., 1981. Structural and immunochemical studies on the phytotoxic peptidorhamnomanann of Ceratocystis ulmi. Plant. Physiol., 67, 1208-1213.

Riffaud J.L., Cornu D., 1981. Utilisation de la culture in vitro pour la multiplication de merisiers adultes (Prunus avium L.) sélectionnés en forêt. Agronomie, 1, 633-640.

SRISkandarajah S., Mullins M.G., NaIR Y., 1982. Induction of adventitious rooting in vitro in difficult-to-propagate cultivars of apple. Plant Sci. Lett., 24, 1-9.

Takal S., Richards W.C., 1978. Cerato-ulmin, a wilting toxin of Ceratocystis ulmi : isolation, and some properties of cerato-ulmin from the culture of C. ulmi. Phytopathol. Z., 91, 129-146.

ZimmermanN R.H., 1980. Proceedings of the conference on nursery production of fruit plants through tissue culture: application and feasibility. Publié par l'Université du Maryland, Betsville, $106 \mathrm{p}$. 